\title{
O fluxo de capital para as economias emergentes e o grau de desenvolvimento do sistema financeiro
}

\author{
Katia Rocha \\ Técnica de Planejamento e Pesquisa - Instituto de Pesquisa Econômica Aplicada (IPEA) \\ Endereço: AV. Presidente Antônio Carlos 51/17th - Castelo - Rio de Janeiro/RJ \\ CEP: 20020-010 - E-mail: katia.rocha@ipea.gov.br
}

Ajax Moreira

Coordenador de Economia Financeira - Instituto de Pesquisa Econômica Aplicada (IPEA)

Endereço: AV. Presidente Antônio Carlos 51/17th - Castelo - Rio de Janeiro/RJ

CEP: 20020-010 - E-mail: ajax.moreira@ipea.gov.br

\begin{abstract}
Marcos Silveira
Técnico de Planejamento e Pesquisa - Instituto de Pesquisa Econômica Aplicada (IPEA)

Endereço: AV. Presidente Antônio Carlos 51/17th - Castelo - Rio de Janeiro/RJ

CEP: 20020-010 - E-mail: marcos.silveira@ipea.gov.br
\end{abstract}

Recebido: 28/10/2014. Aceite: 07/03/2017.

\section{Resumo}

O presente artigo tem como objetivo analisar o papel do sistema financeiro doméstico como mitigador de eventos de paradas súbitas e condicionante de fluxos de capital de um grupo de 14 economias emergentes no período de 1999-2013, em especial em face de cenários externos desfavoráveis como aumento dos juros internacionais e aversão ao risco global. Os países analisados - Argentina, Brasil, Chile, Colômbia, Indonésia, Malásia, México, Peru, Filipinas, Rússia, África do Sul, Tailândia, Turquia e Ucrânia representavam, em agosto de 2014, aproximadamente $80 \%$ do índice EMBIPLUS e $60 \%$ do índice EMBIGLOBAL. Procurou-se distinguir o efeito sobre diversos tipos de fluxos de capital: Fluxo de entrada de Investimento Externo Direto, Fluxo de entrada de Investimento em Carteira e Fluxo Líquido, com análise de robustez para período desde 1990 e aumento do número de países emergentes. O principal resultado suporta evidências que o fortalecimento do sistema financeiro doméstico - medido com a capitalização bursátil em proporção do PIB propicia aumento dos fluxos de entrada de capital de todos os tipos de fluxos em todos os modelos analisados, reduzindo a probabilidade de ocorrência de parada súbita do fluxo de Investimento Externo Direto e em alguns casos do fluxo de Carteira, o que motiva políticas públicas que incentivem o desenvolvimento e fortalecimento deste setor. Estimativas também destacam o papel dos fundamentos domésticos, tão importantes quanto os fatores globais nos condicionantes de fluxo de capital e na ocorrência paradas súbitas. Este fato motiva políticas públicas que incentivem o fortalecimento e desenvolvimento das instituições nos países emergentes, e ressalta o papel dos governos na captação de recursos externos.

\section{Palavras-Chave}

Economias emergentes. Fluxo de capital. Parada súbita. Desenvolvimento do sistema financeiro doméstico. 


\begin{abstract}
The objective of this is study is to assert the role of domestic financial system as mitigating of sudden stops episodes and driver of capital flows in a group of 14 emerging economies in the period of 1999-2013, especially in face of unfavorable external environment such as high international interest rate or global risk aversion. The countries analyzed - Argentina, Brazil, Chile, Colombia, Indonesia, Malaysia, Mexico, Peru, Philippines, Russia, South Africa, Thailand, Turkey and Ukraine, represented in August 2014 roughly 80\% of the EMBIPLUS and $60 \%$ of EMBIGLOBAL. We work with distinct types of capital flows: Inflow of Foreign Direct Investment, Inflow of Portfolio and Net Capital, and conduct robustness check considering data since 1990 and increasing the number of emerging countries. The result supports evidence that improvement of domestic financial system allows an increase of capital flow and a decrease of sudden stops probability. Estimates also indicate that domestic fundamentals are as important as global factors in explaining capital flows and sudden stops episodes. This fact supports public policies that improve the development and strength of domestic financial system in emerging economies and stress the government role in attracting external capital.
\end{abstract}

\title{
Keywords
}

Emerging economies. Capital flow. Sudden stops. Strength of domestic financial system.

\section{JEL Classification}

F01. F20. F32.

\section{Introdução}

O debate sobre fluxo de capital para economias emergentes costuma ser um tópico sensível para a pauta dos gestores de políticas públicas, trazendo diversos desafios relacionados ao gerenciamento de política macroeconômica, e na melhor forma de se apropriar dos benefícios relacionados à entrada do fluxo de capital, limitando os riscos de instabilidade financeira e macroeconômica.

Desde 2003, o cenário econômico externo favorável, capitaneado pela alta liquidez internacional, juntamente com a melhora dos fundamentos dos mercados emergentes, proporcionou grande entrada de fluxo líquido de capital para os países emergentes, sendo apenas interrompido pela crise das hipotecas americanas em meados de 2007, mas com retomada logo em seguida a partir de 2009 até 2010. No entanto, entre 2010 e 2015, houve grande queda dos fluxos de entrada para estes países como um todo, como aponta o relatório do World Economic Outlook (2016). Entre os determinantes dessa queda segundo o relatório, destaca-se principalmente a expectativa de menor crescimento econômico 
dos emergentes, menores preços de commodities, e a possibilidade do fim do ciclo de política monetária expansiva adotada pelas economias desenvolvidas. A partir de um cenário de menor liquidez internacional aliado ao aumento gradativo da atratividade dos ativos em economias desenvolvidas, os fundamentos de economias emergentes (fatores locais) estão tendo maior importância como direcionadores do fluxo de capital. Economias com fracos fundamentos e maiores vulnerabilidades têm sofrido com menores fluxos de capital, encontrando-se mais expostas ao mercado.

O presente estudo tem como objetivo verificar a importância do desenvolvimento do sistema financeiro doméstico como determinante de paradas súbitas e condicionantes do fluxo de capital em um grupo de economias emergentes no período de 2000 até 2013. Os países emergentes analisados: Argentina, Brasil, Chile, Colômbia, Indonésia, Malásia, México, Peru, Filipinas, Rússia, África do Sul, Tailândia, Turquia e Ucrânia representaram, em agosto de 2014, aproximadamente $80 \%$ do índice EMBIPLUS e 60\% do índice EMBIGLOBAL, índices de referência do banco J.P.Morgan. ${ }^{1}$

O principal resultado suporta evidências de que o fortalecimento do sistema financeiro doméstico - medido com a capitalização bursátil em proporção do PIB - propicia aumento dos fluxos de entrada de capital de todos os tipos de fluxos em todos os modelos analisados, reduzindo a probabilidade de ocorrência de parada súbita do fluxo de Investimento Externo Direto e em alguns casos do fluxo de Carteira, motivando desta forma políticas públicas que incentivem o desenvolvimento e fortalecimento deste setor.

O presente texto diferencia-se dos demais ao focar no papel do sistema financeiro doméstico sobre diferentes tipos de fluxo de entrada de capital, e sobre a chance de mudança abrupta deste fluxo. Em particular procuramos distinguir tal efeito sobre diversos tipos de fluxos de capital: i) Fluxo de entrada de Investimento Externo Direto (IED), ii) Fluxo de entrada de Investimento em Carteira (ações, títulos, derivativos e outros investimentos) e iii) Fluxo Líquido (entrada e saída de todos os tipos de capital). Neste sentido, cabe mencionar que praticamente toda a literatura empírica sobre eventos de parada súbita, incluindo os influentes trabalhos de Calvo, Izquierdo e Mejía (2004, 2008), tem

1 Maiores detalhes em J.P.Morgan (2004a, 2004b). Selecionamos países que consideramos semelhantes ao Brasil que tem polupação entre 15 e 250 milhões de habitantes e que não pertencem à zona do euro. 
como objeto de análise a interrupção do fluxo agregado de capital, sem discriminar entre seus diferentes componentes. ${ }^{2}$ Faucette, Rothenberg e Warnock (2005), por exemplo, analisam o comportamento individual de cada tipo de fluxo de capital durante paradas súbitas do fluxo agregado, e não por tipo de fluxo. Até o momento, portanto, não há registro de uma pesquisa sobre os determinantes específicos dos eventos extremos de cada rubrica da conta financeira, a partir da estimação de um modelo econométrico cuja variável dependente seja um indicador de eventos ocorridos exclusivamente nos fluxos desta rubrica.

O trabalho contribui para o preenchimento desta lacuna, cujo esclarecimento é crucial para o desenho de uma política eficiente de controle de capitais. Não é difícil suspeitar que os fluxos das diferentes modalidades de capital abrigadas dentro da conta financeira respondam diferentemente ao estado da economia doméstica e às flutuações do cenário internacional. É fato notório que o investimento externo direto tem um horizonte de longo prazo e é calcado em considerações estratégicas, enquanto o investimento em carteira é resultado da exploração no curto prazo de oportunidades de arbitragem entre mercados de capitais nacionais. Podem ser ressaltadas muitas outras distinções quanto à natureza e finalidade das diferentes rubricas da conta financeira, resultando em um fluxo líquido de capital externo bastante heterogêneo no que tange à maturidade, ao risco, ao emissor, à liquidez e a outras características relevantes de seus ativos financeiros.

A atual estrutura do Balanço de Pagamentos Internacionais adotada pelos países segue uma orientação do Fundo Monetário Internacional FMI estabelecida no documento Balance of Payments and International Investment Position Manual (2013), e registra as transações econômicas de determinado país com o exterior (residentes e não residentes). ${ }^{3}$

2 Sula (2010) exclui o investimento externo direto do fluxo agregado em decorrência de sua maior estabilidade relativa.

3 As contas são agrupadas segundo suas categorias (reais e financeiras) e segundo seus fatos geradores (comércio de mercadorias, prestação de serviços, transferências e movimentos de capital, nas formas de financiamentos e de investimentos diretos). Sua estrutura é definida a partir da natureza das transações, que se agrupam em duas grandes categorias de contas - as transações correntes, conta capital e conta financeira. Em especial, a conta financeira registra fluxos decorrentes de transações com ativos e passivos financeiros entre residentes e não residentes, sendo dividida em quatro grupos:1) investimento direto; 2) investimentos em carteira; 3) derivativos; e 4) outros investimentos. Cada grupo é desdobrado em ativos e passivos, ou seja, fluxos envolvendo ativos externos detidos por residentes no Brasil (valores negativos) e outro para registrar a emissão de passivos por residentes, cujo credor é não residente (valores positivos). O fluxo de capital líquido equivale à soma de todos os itens da conta financeira ativa e passiva, com valor positivo significando entrada líquida de capital. 
Optamos por analisar os fluxos de entrada uma vez que seus determinantes diferenciam-se dos fluxos de saída e dependem da percepção do risco e da rentabilidade esperada de um agente não residente no país. Ressalta-se que os eventos no fluxo de uma rubrica não necessariamente coincidem com os eventos em uma outra rubrica, uma vez que cada modalidade de capital pode responder a um conjunto particular de fatores.

$\mathrm{Na}$ literatura de fluxo de capital, muitos estudos já abordaram questões sobre os determinantes locais e globais (push and pull effects) dos fluxos de capitais para economias emergentes. Dentre os trabalhos seminais nesta linha de pesquisa encontram-se Calvo, Leiderman e Reinhart (1993, 1996) e Fernandez-Arias (1996). Entre os trabalhos após a crise das hipotecas em 2008, destacam-se BIS (2009), Forbes e Warnock (2011), Milesi-Ferretti e Tille (2011), Bekaert et al. (2011) e Fratzscher (2012). Dentre os resultados comuns temos a ênfase dos fatores globais, em especial da liquidez e aversão ao risco internacional como determinantes do fluxo de capital e a heterogeneidade de resposta entre os tipos de fluxo e país afetado. De forma geral, há consenso de que a importância dos fatores globais cresce em períodos de maior instabilidade financeira internacional, sendo ainda preponderante. Fatores locais como fundamentos, expectativas de crescimento das economias emergentes ou variáveis estruturais como qualidade das instituições tornam-se gradualmente mais importantes nos ciclos financeiros favoráveis, sendo algumas vezes capazes de explicar a heterogeneidade da resposta entre os emergentes.

Por sua vez, a extensa literatura sobre os determinantes dos eventos extremos de paradas súbitas de capitais também enfatiza a diferenciação entre fatores globais (push factors) e locais (pull factors). Forbes e Warnock (2011) analisam os efeitos de variáveis globais, domésticas e relativas a contágio sobre a probabilidade de ocorrência de movimentos extremos de capital em uma amostra de países emergentes e desenvolvidos. Calvo, Izquierdo e Mejía (2008) concluem que, embora eventos sistêmicos de parada súbita sejam detonados por choques exógenos, sua magnitude e suas consequências dependem das condições internas das economias domésticas, em particular a existência de desequilíbrios externos, o grau de dolarização dos passivos dos agentes domésticos e a sensibilidade da taxa de câmbio real à interrupção da entrada de capital externo. 
Enfatizando o papel do sistema financeiro doméstico citamos Broner e Rigobon (2004), Alfaro et al. (2005), Global Financial Stability Report (2007), Neumann et al. (2009), Broto et al. (2011) e Rocha e Moreira (2013). ${ }^{4}$ Os principais resultados destacam uma redução potencial da volatilidade do fluxo de entrada de diversos tipos de capital mediante a adoção de políticas de desenvolvimento do sistema financeiro doméstico, muito embora sublinhem a dificuldade de encontrar políticas específicas que, regra geral, possam reduzir a volatilidade de todos os tipos de fluxo de capital.

A próxima seção apresenta a metodologia para definição de períodos de parada súbita; a seção 3 apresenta a metodologia para determinantes de parada súbita e condicionantes de fluxo de capital; a seção 4 apresenta os resultados, enquanto a seção 5 conclui.

\section{Identificação dos períodos de parada súbita de capital}

Três regularidades empíricas podem ser extraídas dos eventos extremos de parada súbita: $i$ ) os eventos não são infrequentes, afligindo boa parte das economias emergentes pelo menos uma vez por década; ii) os eventos são o resultado de decisões financeiras de investidores externos, não residentes, devendo então ser interpretados como choques externos sobre a conta financeira da economia doméstica; e iii) os eventos são acompanhados por uma forte contração do nível de atividade e investimento e/ou uma forte depreciação cambial. É a partir destas evidências que a alteração abrupta do fluxo líquido de capitais (sudden stops) é discutida por Calvo, Izquierdo e Mejia (2004, 2008), o qual propõe uma metodologia bem difundida para identificar tais eventos.

Em linhas gerais, dada a série histórica do fluxo de entrada de capitais externos (não residentes) de uma rubrica da conta financeira de um país, esta metodologia acusa a ocorrência de uma parada súbita em um dado trimestre, quando a redução do fluxo em relação ao trimestre anterior situa-se dois desvios padrões abaixo da média das variações

4 Ressaltamos a diferença entre Rocha e Moreira (2013) e o presente texto, sendo o primeiro focado apenas nos determinantes da volatilidade (segundo momento) dos fluxos de capital, enquanto neste trabalho são analisados os determinantes de parada súbita e da média (primeiro momento) dos respectivos fluxos. 
trimestrais do fluxo no passado recente. Para todo trimestre, o cálculo da média e do desvio padrão dos fluxos no passado recente fazem uso de uma janela de vinte trimestres anteriores. Os principais resultados encontrados neste trabalho são robustos a uma mudança no tamanho da janela.

Procedimentos alternativos para identificação de eventos extremos, em particular paradas súbitas, são encontrados na literatura empírica. Calvo, Izquierdo e Mejía (2004) impõem sobre o procedimento anterior o requerimento adicional de que a interrupção do ingresso de capital externo em um país seja acompanhada por uma queda de seu produto agregado. Isto é feito com o propósito de excluir da amostra os eventos em que a redução do superávit da conta financeira foi causada por uma reversão estrutural da trajetória da conta corrente ou por uma melhoria nos termos de troca do comércio exterior, os quais não se configuram eventos típicos de paradas súbitas decorrentes do fechamento repentino e conjuntural das fontes de financiamento externo. Outros procedimentos de identificação de paradas súbitas podem ser motivados por um conceito diferente de volatilidade dos fluxos de capitais. Por exemplo, Edwards (2004) identifica um evento quando a redução do saldo da conta financeira, em relação ao período anterior, é superior a uma dada proporção do PIB corrente. Este procedimento acusa um número maior de eventos nos países com fluxos externos relativamente mais voláteis, ignorando, assim, o fato de que uma reversão no superávit de uma conta financeira altamente volátil é bem menos representativa como parada súbita que uma reversão de mesma magnitude em uma conta pouco volátil. Por sua vez, um procedimento baseado no desvio padrão das variações do fluxo, como o adotado em Calvo (1998), acusa um número considerável de eventos, mesmo em países cujos fluxos são relativamente estáveis pelos padrões internacionais.

A fim de minimizar o risco de identificação equivocada de eventos de parada súbita, Guidotti, Sturzenegger e Villar (2004) e Cowan et al. (2008) acusam a ocorrência de um evento somente quando são observadas as condições de ambos os procedimentos acima descritos. De qualquer forma, é desnecessário dizer que a escolha do procedimento mais adequado é ainda uma questão controversa na literatura. Este trabalho lança mão do procedimento em Calvo, Izquierdo e Mejía (2004) possibilitando, assim, a identificação de eventos em um número maior de países e em um período mais extenso. Além disso, este procedimento é mais amplamente difundido na literatura, o que torna os resulta- 
dos deste trabalho comparáveis aos de outros trabalhos correlatos. Para cada rubrica da conta financeira, o trabalho identifica separadamente os eventos de parada súbita nos fluxos de capitais.

Caso os eventos extremos ocorram simultaneamente nos fluxos de todas as modalidades de capital, ou mesmo no caso menos extremo de uma alta correlação entre eles, pouca informação adicional seria provida por identificá-los separadamente no fluxo de cada rubrica da conta financeira. Isto porque as variáveis dependentes indicadoras dos eventos em fluxos agregados ou desagregados seriam fortemente correlacionadas.

As Tabelas abaixo apresentam algumas estatísticas dos eventos de paradas subidas. A Tabela 1 apresenta a proporção dos trimestres com eventos de parada súbita por país e tipo de fluxo. Pode-se verificar que o Brasil apresenta parada súbita em 21\% dos trimestres analisados para o fluxo de Carteira, 32\% para Investimento Externo Direto e 17\% para fluxo líquido total.

Tabela 1 - Parada Súbita por país e tipo de fluxo (proporção dos trimestres)

\begin{tabular}{|l|r|r|r|}
\hline & Fluxo Líquido & \multicolumn{1}{|c|}{ Carteira } & \multicolumn{1}{|c|}{ Investimento Externo Direto } \\
\hline Argentina & 0.52 & 0.29 & 0.24 \\
\hline Brasil & 0.17 & 0.21 & 0.32 \\
\hline Chile & 0.40 & 0.10 & 0.32 \\
\hline Colômbia & 0.13 & 0.16 & 0.18 \\
\hline Indonésia & 0.48 & 0.23 & 0.34 \\
\hline Malásia & 0.66 & 0.53 & 0.24 \\
\hline México & 0.17 & 0.14 & 0.24 \\
\hline Peru & 0.17 & 0.31 & 0.18 \\
\hline Filipinas & 0.43 & 0.27 & 0.25 \\
\hline Rússia & 0.67 & 0.19 & 0.29 \\
\hline África do Sul & 0.21 & 0.38 & 0.22 \\
\hline Tailândia & 0.54 & 0.24 & 0.29 \\
\hline Turquia & 0.19 & 0.24 & 0.30 \\
\hline Ucrânia & 0.36 & 0.20 & 0.26 \\
\hline Total & 0.36 & 0.24 & 0.26 \\
\hline
\end{tabular}

Fonte: Elaboração Própria. 
Consideramos a concomitância dos eventos de parada súbita de um certo tipo de fluxo entre países, e a concomitância destes eventos entre fluxos de um certo país. A Tabela 2 apresenta o resultado do teste da hipótese de independência, utilizando o teste exato de Fisher (1922), realizado para cada um dos indicadores de parada súbita separadamente. A hipótese nula é que a ocorrência de crise em um país, indicado na linha, não depende da ocorrência de crise nos demais países da amostra. Na tabela está indicado para cada país e tipo de fluxo o resultado do teste denotando com o sinal $\left({ }^{*}\right)$ os casos em que foi rejeitada a hipótese de independência. Observa-se que para a maioria dos casos não rejeitamos a dependência entre os países, o que sugere a existência de uma causa comum, ou seja, a crise tende a ocorrer de forma concomitante entre os países.

Tabela 2 - Concomitância das Paradas Súbitas entre Países

\begin{tabular}{|l|c|c|c|}
\hline & Fluxo Líquido & Carteira & Investimento Externo Direto \\
\hline Argentina & ${ }^{*}$ & ${ }^{*}$ & ${ }^{*}$ \\
\hline Brasil & ${ }^{*}$ & ${ }^{*}$ & ${ }^{*}$ \\
\hline Chile & ${ }^{*}$ & ${ }^{*}$ & ${ }^{*}$ \\
\hline Colômbia & ${ }^{*}$ & ${ }^{*}$ \\
\hline Indonésia & & ${ }^{*}$ & ${ }^{*}$ \\
\hline Malásia & ${ }^{*}$ & ${ }^{*}$ \\
\hline México & ${ }^{*}$ & ${ }^{*}$ & \\
\hline Peru & ${ }^{*}$ & ${ }^{*}$ & ${ }^{*}$ \\
\hline Filipinas & & ${ }^{*}$ & ${ }^{*}$ \\
\hline Rússia & ${ }^{*}$ & ${ }^{*}$ & ${ }^{*}$ \\
\hline África do Sul & ${ }^{*}$ & ${ }^{*}$ & ${ }^{*}$ \\
\hline Tailândia & ${ }^{*}$ & ${ }^{*}$ & ${ }^{*}$ \\
\hline Turquia & ${ }^{*}$ & & \\
\hline Ucrânia & &
\end{tabular}

O sinal $\left(^{*}\right)$ indica que foi rejeitada ao nível de 1\%, a hipótese de que as crises do país indicado na linha sejam independentes das crises ocorridas nos demais 13 países da amostra.

Fonte: Elaboração Própria. 
A concomitância de eventos entre fluxos é avaliada na Tabela 3, que documenta o número de trimestres/países para os quais ocorreu a condição de crise e não crise segundo os pares de fluxos. ${ }^{5}$ Aqui também foi realizado o teste exato de Fisher (1922) para testar a hipótese de que as crises segundo pares de indicadores são independentes entre si. O teste rejeitou, com o nível de $1 \%$, a hipótese de independência de parada súbita entre os fluxos, i.e., quando ocorre uma crise em um tipo de fluxo há chances elevadas de ocorrência de crise em outro tipo de fluxo, o que sugere novamente o fator comum como variável explicativa das crises. Por exemplo, de 213 eventos de crise no Investimento Externo Direto, ocorreram 70 eventos de crise no fluxo de Carteira. De outra forma, de 190 eventos de crise no fluxo de Carteira, ocorreram 70 eventos de crise no Investimento Externo Direto.

Tabela 3 - Concomitância das Paradas Súbitas entre Fluxos

\begin{tabular}{|c|c|c|c|c|c|c|}
\hline & \multicolumn{3}{|c|}{ Investimento Externo Direto } & \multicolumn{3}{c|}{ Fluxo Líquido } \\
\hline & Não crise & Crise & Total & Não crise & Crise & Total \\
\hline Carteira & 466 & 143 & 609 & 419 & 184 & 603 \\
\hline Não crise & 120 & 70 & 190 & 100 & 90 & 190 \\
\hline Crise & 586 & 213 & 799 & 519 & 274 & 793 \\
\hline Total & \multicolumn{7}{|l|}{} & 395 & 196 & 591 \\
\hline IED & & & & 128 & 86 & 214 \\
\hline Não crise & & & & 523 & 282 & 805 \\
\hline Crise & &
\end{tabular}

Fonte: Elaboração Própria.

Os resultados apresentados mostram que: i) o fluxo de capitais para os países emergentes está sujeito a alterações bruscas periódicas, chamadas de parada súbita ou crise; ii) as crises ocorrem de forma concomitante entre os países; iii) as crises não ocorrem de forma concomitante entre os tipos de fluxo.

5 Como a disponibilidade de dados não é igual entre os fluxos, a linhas de Total da tabela para cada par de fluxos não é sempre a mesma. 


\section{Metodologia}

Uma forma parcimoniosa de relacionar o fluxo de capitais (Y) com as variáveis locais e globais $(\mathrm{L}, \mathrm{G})$ é admitir que o fluxo de capitais está sujeito à crise ${ }^{6}$ como indicado na Equação 1 e que a ocorrência da crise também é explicada com as mesmas variáveis $(L, G)$ como na Equação 2 , onde $I_{i t}$ é um dos indicadores de parada súbita já definidos, $G_{t}$ o vetor de variáveis globais no trimestre $t$ e $L_{i t}$ o vetor de variáveis locais do país $i$ no trimestre.

$$
\begin{aligned}
& \mathrm{E}\left(\mathrm{Y}_{\mathrm{it} \mid} \mid \mathrm{G}_{\mathrm{t}}, \mathrm{L}_{\mathrm{it}}\right)=\alpha_{\mathrm{i}}+\beta \mathrm{G}_{\mathrm{t}}+\gamma \mathrm{I}_{\mathrm{it}}+\delta \mathrm{L}_{\mathrm{it}} \\
& \operatorname{Prob}\left(\mathrm{I}_{\mathrm{it}}=1 \mid \mathrm{G}_{\mathrm{t}} \mathrm{L}_{\mathrm{it}}\right)=\Phi\left(\alpha_{\mathrm{i}}+\beta \mathrm{G}_{\mathrm{t}}+\delta \mathrm{L}_{\mathrm{it}}\right)
\end{aligned}
$$

Os países estão igualmente expostos às condições internacionais do mercado de capital, no entanto, o fluxo de capitais para alguns países emergentes é mais sensível a alterações das condições externas, sendo que em alguns casos pode ocorrer ainda uma alteração abrupta de parada súbita. Naturalmente, são as condições domésticas que explicam este diferencial de sensibilidade.

A Equação 1 é uma forma parcimoniosa de lidar com o efeito da crise, supondo que na crise os países tem uma alteração do fluxo - como proporção do PIB - comum medida com $(\gamma),{ }^{7}$ e explicita o efeito das variáveis globais e locais $(\mathrm{G}, \mathrm{L})$ sobre cada tipo de fluxo. Considerando que os países emergentes são pequenos diante do mercado global de capitais, podemos admitir que as variáveis globais condicionam as locais, mas não são condicionadas por estas. Sob esta hipótese a diferença entre a proporção da variância explicada pelo modelo $E\left(Y_{i t} \mid G_{t}, L_{i t}\right)$ e $E\left(Y_{i t} \mid G_{t}\right)$ é uma medida da importância das variáveis domésticas na explicação do fluxo.

A estimativa do efeito das variáveis globais e locais $(G, L)$ sobre a ocorrência da crise foi realizada segundo três modelos representados na Eq.(2): i) o modelo logit para painel (rotina xtlogit do Stata) que inclui um efeito fixo por país mas é menos eficiente, ii) o modelo probit para painel (rotina xtprobit do Stata), que inclui um efeito aleatório por país,

6 Este indicador é uma função dos valores passados da variável dependente.

7 Nesta forma, admitimos que a resposta do fluxo às explicativas não se altera no estado de crise, e que a alteração do nível do fluxo no período da crise é a mesma em todos os países. Esta especificação pode ser flexibilizada de várias formas, mas a disponibilidade dos dados dificulta a estimação de formas mais flexíveis. 
e finalmente, iii) o modelo probit com variância por cluster de país, que lida de forma menos robusta com características idiossincráticas de cada país, mas tem a vantagem de requerer amostras menores e possibilitar o cálculo da taxa de acerto na identificação das crises. Na falta de uma motivação conceitual para a escolha de um destes modelos, analisamos os três modelos e avaliamos a robustez dos resultados. Vale ressaltar que a forma funcional do modelo xtlogit é distinta da forma dos demais, e que portanto os coeficientes estimados deste modelo não são diretamente comparáveis aos dos demais.

\section{Resultados}

A hipótese básica do modelo adotado é que os coeficientes das variáveis explicativas são comuns a todos os países. Isto recomenda que os países selecionados sejam, de alguma forma, semelhantes entre si. Os países emergentes foram selecionados descartando países muito grandes (China e Índia) - e com menos de 10 milhões de habitantes. Descartamos também países emergentes da Zona do Euro. Considerando estes critérios resultou a lista de 14 países indicados na Tabela 4 para o período 1999-2013.

Tabela 4 - Países Selecionados

\begin{tabular}{|l|r|r|r|l|r|r|r|}
\hline & PIB/POP & PIB & POP & & PIB/POP & PIB & POP \\
\hline Argentina & 6859 & 284 & 39 & Peru & 942 & 27 & 28 \\
\hline Brasil & 1709 & 327 & 188 & Filipinas & 397 & 37 & 87 \\
\hline Chile & 2290 & 40 & 16 & Rússia & 1881 & 284 & 144 \\
\hline Colômbia & 1100 & 50 & 44 & África do Sul & 5178 & 263 & 49 \\
\hline Indonésia & 476 & 118 & 228 & Tailândia & 860 & 59 & 65 \\
\hline Malásia & 1681 & 47 & 26 & Turquia & 1899 & 137 & 68 \\
\hline México & 8052 & 934 & 112 & Ucrânia & 578 & 28 & 46 \\
\hline
\end{tabular}

Nota: PIB em bilhões de US dólares, POP (População em milhões de habitantes).

Valores médios do período de 1999-2013.

A fonte de dados utilizados são as contas financeiras do balanço de pagamentos publicadas pelo International Finance Statistics - IFS do FMI em bases trimestrais, e de forma desagregada segundo as principais 
contas: i) Fluxo de entrada de Investimento Externo Direto, ii) Fluxo de entrada de Investimento em Carteira (Ações + Títulos + Derivativos + Outros Investimentos) e iii) Fluxo Líquido (entrada e saída de todos os tipos de capital).

Para responder a questão da importância do desenvolvimento do sistema financeiro doméstico na determinação de paradas súbitas e do fluxo de capital, utilizamos dois indicadores: i) capitalização bursátil sobre PIB, ii) crédito privado sobre PIB, ambas variáveis disponíveis no Banco Mundial. ${ }^{8}$

Os fatores globais adotados correspondem à medida de aversão ao risco - VIX ${ }^{9}$ e a taxa de juros americana de 10 anos - USTRY como uma medida de liquidez global. As variáveis explicativas locais adotadas neste estudo que correspondem a fundamentos macroeconômicos do país. A maioria dos dados está disponível no banco de dados do IFS-FMI, e em outras fontes indicadas no texto. Dentre os fatores locais adotamos a taxa real de crescimento econômico, inflação, juro real, abertura comercial (soma das exportações e importações sobre PIB) e reservas financeiras totais sobre importação. As variáveis locais foram incorporadas no modelo com a defasagem de um trimestre para aquelas apuradas trimestralmente, e de 1 ano para aquelas apuradas em termos anuais. Este cuidado evita críticas quanto à endogeneidade destas variáveis. O modelo também supõe que as respostas sejam invariantes ao longo tempo, novamente uma forma de lidar com esta questão é limitar a amostra ao período mais recente - pós 1999.

8 O banco mundial desenvolve indicadores (Global Financial Development) do sistema financeiro (instituições financeiras e mercado financeiro) de 203 países incluindo medidas de: (i) tamanho; ii) grau de eficiência; e iii) estabilidade. Disponível em: http://data.worldbank.org/data-catalog/ global-financial-development. Segundo Levine (2005) um grande número de evidências sugere que o sistema financeiro doméstico, incluindo instituições financeiras (bancos e seguradoras) e mercado financeiro (ações, títulos e derivativos), exerce uma grande influência no desenvolvimento econômico, estabilidade econômica e diminuição do nível de pobreza. O aumento da capitalização bursátil está relacionado com o aumento do número de empresas incorporadas à bolsa e também com o aumento do valor dos ativos. Sendo que este último é uma medida da expectativa do mercado quanto ao futuro da economia. Isto faz com que a interpretação do efeito desta variável tenha estas duas dimensões. Da mesma forma o aumento do crédito privado também está relacionado com duas dimensões, a penetração do mercado financeiro na economia, e com a expectativa quanto ao futuro que induz os agentes a se endividarem.

9 O índice VIX é comumente utilizado como proxy da aversão ao risco de mercado. Foi introduzido em 1993 pela Chicago Board Options Exchange (2009), e consiste na volatilidade diária implícita nas opções sobre o índice S\&P 500 para os próximos 30 dias considerando diversas ponderações sobre preços de opções de diversos preços de exercício. 
Para avaliar a robustez do resultado os modelos foram estimados para 3 amostras: 1) a que considera a maior homogeneidade - seleção dos 14 países emergentes e período pós 1999 - que denotamos por Caso Base; 2) flexibilizando o período de tempo e com isto considerando todos os dados disponíveis que começam em 1990 que denotamos por Período Pós 1990; e finalmente incorporando todos os 27 países emergentes da amostra Total Emergentes. ${ }^{10}$ Vale mencionar que algumas variáveis não estão disponíveis para todos os países e períodos de tempo, de forma que as amostras definidas não aumentam de tamanho segundo os múltiplos de países e períodos adicionados.

\subsection{Determinantes dos eventos de parada súbita de capital}

A Tabela 5 apresenta os resultados e significância dos coeficientes estimados da Equação 2 para o caso base (seleção de 14 países e o período pós 1999). Os resultados para as demais amostras estão no Apêndice A.

Tabela 5 - Determinantes de Parada Súbita ${ }^{11}$

\begin{tabular}{|c|c|c|c|c|c|c|c|c|c|}
\hline & \multicolumn{3}{|c|}{ Fluxo Líquido } & \multicolumn{3}{|c|}{ Carteira } & \multicolumn{3}{|c|}{ Investimento Externo Direto } \\
\hline Caso Base & xtprobit & probit & xtlogit & xtprobit & probit & xtlogit & xtprobit & probit & xtlogit \\
\hline \multicolumn{10}{|c|}{ Variáveis Globais } \\
\hline VIX & $2.63^{* *}$ & $2.37^{* *}$ & $4.41^{* *}$ & $6.67^{\star \star}$ & $6.62^{\star \star}$ & $11.84^{* *}$ & $4.69^{\star *}$ & $4.61^{* *}$ & $8.07^{\star *}$ \\
\hline USTRY10 & $8.81^{*}$ & 9.05 & 11.63 & $10.04^{*}$ & 10.35 & $27.39^{\star *}$ & $14.64^{* *}$ & $13.50^{\star \star}$ & $22.96^{\star \star}$ \\
\hline \multicolumn{10}{|c|}{ Variáveis de Desenvolvimento do Sistema Financeiro Doméstico } \\
\hline $\begin{array}{l}\text { Capitalização } \\
\text { Bursátil }\end{array}$ & -0.27 & -0.01 & -0.61 & -0.11 & 0.03 & -0.36 & $-0.48^{* *}$ & $-0.35^{\star}$ & $-1.78^{\star *}$ \\
\hline Crédito Privado & -0.11 & -0.12 & $-1.23^{*}$ & $0.81^{* *}$ & 0.45 & $4.00^{* *}$ & 0.25 & 0.21 & -1.09 \\
\hline \multicolumn{10}{|c|}{ Variáveis Locais } \\
\hline Inflação & 1.21 & 1.20 & $2.79^{\star \star}$ & $1.63^{*}$ & 1.66 & 1.56 & 0.79 & 0.99 & 1.18 \\
\hline Juros Reais & 0.50 & 0.61 & 1.38 & $2.79^{\star \star}$ & $2.41^{* *}$ & $4.45^{\star \star}$ & $1.57^{\star \star}$ & 1.42 & $3.79^{\star \star}$ \\
\hline Abertura Comercial & 42.11 & $84.02^{* *}$ & -121.53 & 15.26 & 12.17 & 140.40 & 30.63 & $25.32^{*}$ & $196.36^{\star *}$ \\
\hline Crescimento PIB & $-2.73^{\star \star}$ & $-3.23^{\star *}$ & $-4.77^{\star *}$ & $2.67^{*}$ & 2.31 & $6.58^{\star \star}$ & $-6.37^{* *}$ & $-6.18^{\star *}$ & $-12.13^{\star \star}$ \\
\hline Reserva/Importado & 0.95 & 7.82 & -1.19 & 5.81 & 4.82 & 8.10 & 8.28 & 5.53 & $42.35^{\star \star}$ \\
\hline
\end{tabular}

Fonte: Elaboração Própria.

${ }^{10} 27$ países no total que inclui a lista anterior acrescida de: Bolívia, Bulgária, China, Croácia, República Tcheca, Hungria, Índia, Coréia do Sul, Paraguai, Polônia, Singapore, Uruguai e Venezuela.

${ }^{11}$ Os modelos são não lineares e, portanto, estes coeficientes não podem ser interpretados como o efeito da variável sobre a probabilidade. Para isto é necessário calcular o efeito valor marginal. No entanto, sendo este coeficiente significativo, o efeito marginal também o será. 
Os resultados indicam que o aumento da aversão ao risco global e das taxas de juros americanas (redução da liquidez) aumenta a probabilidade de ocorrência de parada súbita em todos os casos. No que tange ao papel do sistema financeiro, observamos que o aumento da capitalização bursátil diminui a probabilidade de ocorrência de parada súbita (probabilidade de crise) de Investimento Externo Direto (IED) em todos os modelos.

De forma a avaliar a robustez desses resultados, apresentamos na Tabela 6 abaixo apenas os coeficientes das variáveis que descrevem o papel do sistema financeiro para todas as amostras de períodos, países e modelos propostos.

Tabela 6 - Desenvolvimento do Sistema Financeiro Doméstico

\begin{tabular}{|c|c|c|c|c|c|c|c|c|c|}
\hline & \multicolumn{3}{|c|}{ Fluxo Líquido } & \multicolumn{3}{|c|}{ Carteira } & \multicolumn{3}{|c|}{ Investimento Externo Direto } \\
\hline & xtprobit & probit & xtlogit & xtprobit & probit & xtlogit & xtprobit & probit & xtlogit \\
\hline \multicolumn{10}{|c|}{ Capitalização Bursátil } \\
\hline 14 países Pós 1990 & -0.26 & 0.12 & $-0.80^{* *}$ & -0.25 & -0.16 & -0.3 & -0.23 & -0.22 & $-1.16^{* *}$ \\
\hline 14 países Pós 1999 & -0.27 & -0.01 & -0.61 & -0.11 & 0.03 & -0.36 & $-0.48^{\star *}$ & $-0.35^{\star}$ & $-1.78^{* *}$ \\
\hline 27 Países Pós 1999 & -0.24 & 0.19 & $-0.67^{* *}$ & $-0.52^{\star *}$ & $-0.32^{*}$ & $-0.91^{* *}$ & $-0.33^{\star *}$ & $-0.21^{*}$ & $-1.27^{* *}$ \\
\hline \multicolumn{10}{|c|}{ Crédito Privado } \\
\hline 14 países Pós 1990 & $-0.70^{* *}$ & -0.35 & $-1.89^{\star *}$ & $1.07^{\star \star}$ & $0.56^{* *}$ & $4.41^{* *}$ & -0.11 & -0.12 & $-1.13^{\star *}$ \\
\hline 14 países Pós 1999 & -0.11 & -0.12 & $-1.23^{*}$ & $0.81^{* *}$ & 0.45 & $4.00^{\star *}$ & 0.25 & 0.21 & -1.09 \\
\hline 27 Países Pós 1999 & -0.24 & -0.12 & -0.59 & $1.27^{\star \star}$ & $0.57^{* *}$ & $3.37^{\star \star}$ & 0.12 & 0.1 & -0.06 \\
\hline \multicolumn{10}{|c|}{ Significativo a $10 \%\left({ }^{*}\right)$ e a $5 \%\left({ }^{* *}\right)$} \\
\hline
\end{tabular}

Fonte: Elaboração Própria. 
Os resultados indicam que:

- O aumento da capitalização bursátil reduz a probabilidade de parada súbita do fluxo de Investimento Externo Direto em todas as amostras e segundo a maioria dos modelos; e do fluxo de Carteira para todos os modelos com a amostra de 27 países e do fluxo Líquido segundo o modelo xtlogit. Vale registrar a semelhança dos coeficientes estimados com os modelos probit e xtprobit;

- O aumento do crédito privado aumenta a probabilidade de parada súbita do fluxo de Carteira para todas amostras e a maioria dos modelos, reduzindo a probabilidade de parada súbita do fluxo Líquido para a amostra pós 1990;

- A forma funcional do modelo e a escolha da amostra não altera o sinal da resposta o que é uma indicação da robustez dos resultados.

\subsection{Determinantes do fluxo de capital}

A Tabela 7 apresenta os resultados obtidos para os determinantes do fluxo obtido pela Equação 1 e a proporção da variância (R2) do fluxo que é explicada com o modelo. Para cada um dos casos, foi utilizada a mesma amostra, incluindo no modelo apenas as variáveis globais, e calculada a mesma estatística denotada por $R 2 \mid \mathrm{G}_{\mathrm{t}}$. Admitindo que as variáveis locais sejam condicionadas, mas não condicionam as variáveis globais, a diferença na capacidade explicativa dos modelos ${ }^{12} E\left(Y_{i t} \mid G_{t}, L_{i t}\right)$ e $E\left(Y_{i t} \mid G_{t}\right)$ pode ser interpretada como a contribuição das variáveis explicativas na explicação da variação do fluxo (linhas 4, 5 e 6 da Tabela). Para avaliação da robustez, o modelo foi estimado com as mesmas 3 amostras de países e períodos mencionadas na seção anterior.

${ }^{12} \mathrm{O}$ modelo foi estimado com a rotina $x$ treg com efeito fixo por país e estimador robusto. 


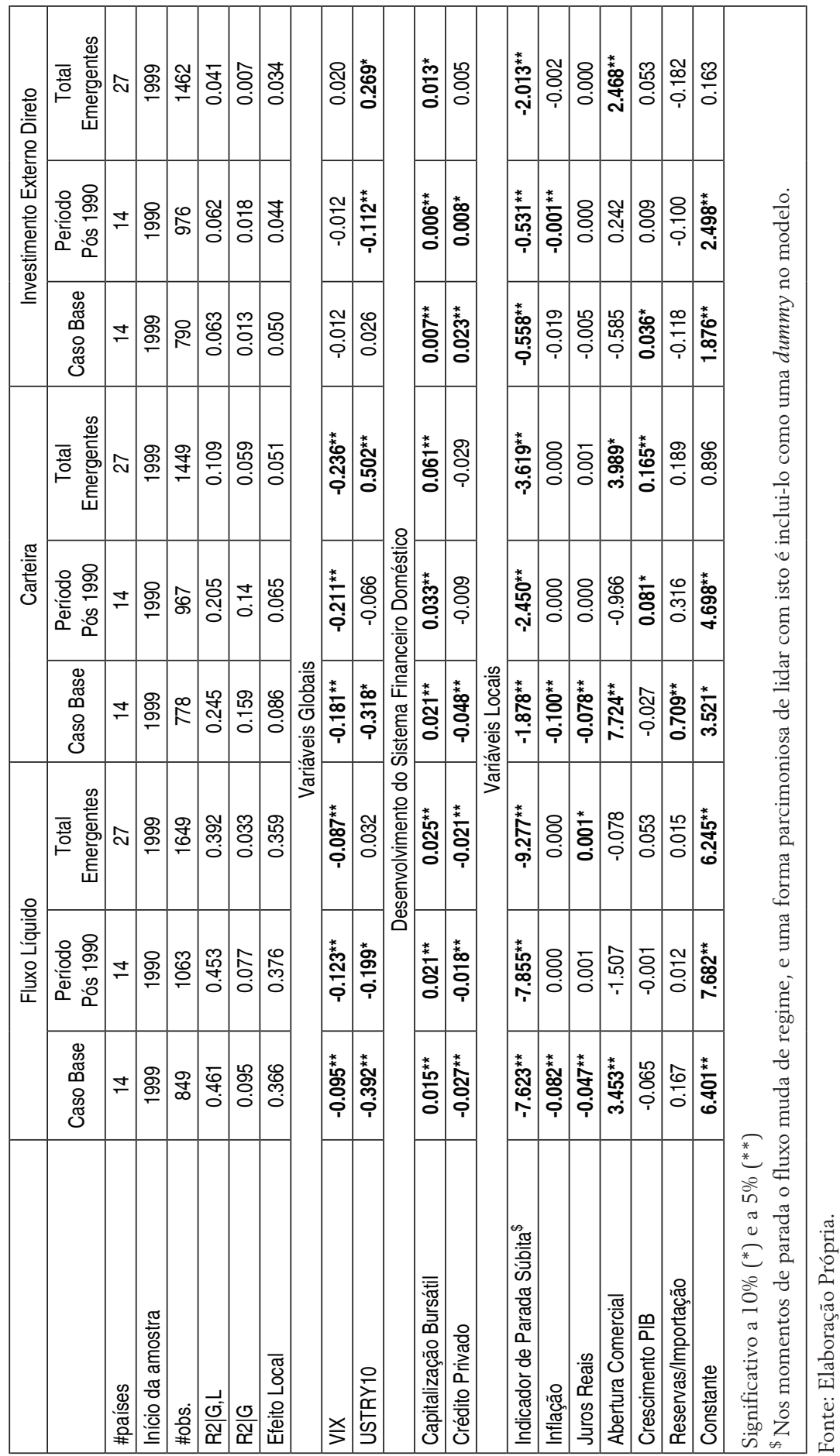


Os resultados indicam que:

- O capital bursátil tem efeito positivo e significativo para todos os fluxos e amostras. Um aumento de $10 \%$ na razão capital bursátil/PIB, implica o aumento de até $0.6 \%$ do Fluxo de Carteira e de até $0.25 \%$ do Fluxo Líquido para todos os emergentes;

- O crédito privado tem efeito positivo para a entrada de investimento direto, e efeito negativo ou estatisticamente igual a zero para os dois outros fluxos, e isto de forma estável para todas as amostras. Um aumento de $10 \%$ na razão crédito privado/PIB, implica o aumento $0.23 \%$ do Fluxo de IED;

- Um aumento de $1 \%$ a.a. nos juros americanos diminui em $0.4 \%$ do PIB a entrada de Fluxo Líquido e Carteira - valor semelhante ao encontrado em Institutional of International Finance (2014). Para IED, essa relação é menor ou não significativa;

- Um amento do coeficiente de aversão ao risco (VIX) da ordem de 10 unidades (um desvio padrão que levaria os valores atuais a um patamar correspondente a média da crise de 2008) diminui em 1 a 2\% do PIB a entrada de Fluxo Líquido e Carteira. Novamente, para IED essa relação é menor ou não significativa;

- O modelo apresenta o melhor desempenho para o caso do Fluxo Líquido, para o qual a média da proporção da variância do fluxo de cada país (R2|G,L) alcança $46 \%$, e o pior desempenho para o IED (ordem de 5\%);

- A diferença entre as médias das proporções de variância do fluxo de cada país dos modelos (R2|G,L)-(R2|G) é uma medida do efeito das condições locais. Novamente o Efeito Local (sexta linha da Tabela) é mais importante para o Fluxo Líquido, para o qual explica até $37 \%$ da variância. De forma consistente o modelo funciona pior para os dois outros fluxos.

\section{Conclusões}

O debate sobre fluxo de capital para economias emergentes costuma ser um tópico sensível para a pauta dos gestores de políticas públicas e, recentemente, versa sobre as consequências de uma possível diminuição da liquidez global com a gradual eliminação dos estímulos econômicos 
americanos, e sobre o papel dos fundamentos domésticos de países emergentes.

O presente estudo está inserido na literatura de determinantes de parada súbita e fluxo de capital, e tem como objetivo analisar o papel do sistema financeiro doméstico como mitigador de eventos de paradas súbitas e condicionante de fluxos de capital de um grupo de 14 economias emergentes no período de 1999-2013, em especial em face de cenários externos desfavoráveis como aumento dos juros internacionais e aversão ao risco global. Os países emergentes analisados - Argentina, Brasil, Chile, Colômbia, Indonésia, Malásia, México, Peru, Filipinas, Rússia, África do Sul, Tailândia, Turquia e Ucrânia, representavam em agosto de 2014 aproximadamente $80 \%$ do índice EMBIPLUS e 60\% do índice EMBIGLOBAL, ambos índices de referência do banco J.P.Morgan.

Procurou-se distinguir o efeito sobre diversos tipos de fluxos de capital: Fluxo de entrada de Investimento Externo Direto, Fluxo de entrada de Investimento em Carteira e Fluxo Líquido, com análise de robustez para período desde 1990 e aumento do número de países emergentes. O principal resultado suporta evidências que o desenvolvimento e fortalecimento do sistema financeiro doméstico - medido com a capitalização bursátil em proporção do PIB - propicia o aumento dos fluxos de entrada de capital de todos os tipos de fluxos em todos os modelos analisados. Em particular, o aumento da capitalização bursátil reduz a probabilidade de ocorrência de parada súbita do fluxo de Investimento Externo Direto e em alguns casos do fluxo de Carteira, o que motiva políticas públicas que incentivem o desenvolvimento e fortalecimento deste setor.

Modelos de painel extraem padrões de resposta de um conjunto de países para determinado período de tempo. Tal padrão admite a homogeneidade de resposta entre países ao longo do tempo. O fato do desenvolvimento do sistema financeiro doméstico ser estatisticamente significativo para explicar diversos tipos de fluxos e a ocorrência de paradas súbitas nestes fluxos para uma gama diversa de países emergentes é digno de nota e ilustra a importância do desenvolvimento do mercado de capitais como um mecanismo estabilizador e de partilha de riscos entre os agentes, motivando desta forma políticas públicas que incentivem o desenvolvimento e fortalecimento deste setor. 
O estudo não rejeita que as crises ocorrem de forma dependente entre os países, o que sugere a existência de fatores comuns, o que é corroborado com o fato de que as variáveis comuns de risco e liquidez global serem significativas em todos os modelos. As estimativas indicam que um aumento de $1 \%$ a.a. nos juros americanos diminui em $0.4 \%$ do PIB a entrada de Fluxo Líquido/PIB e Fluxo de Carteira/PIB - valor semelhante ao encontrado na literatura atual. Com relação ao Investimento Externo Direto (IED), essa relação é menor ou não significativa. Um amento do coeficiente de aversão ao risco (VIX) da ordem de 10 unidades (um desvio padrão) diminui em 1 a 2\% do PIB a entrada de Fluxo Líquido/PIB e Fluxo de Carteira/PIB. Novamente, para IED essa relação é menor ou não significativa.

Finalmente, estimativas também destacam o papel dos fundamentos domésticos, tão importantes quanto os fatores globais nos condicionantes de fluxo de capital e na ocorrência de paradas súbitas, fato que ressalta o papel das instituições na captação de recursos externos.

\section{Referências}

ALFARO, L.; Kalemli-Ozcan, S.; Volosovych, V. Capital Flows in a Globalized World: The Role of Policies and Institutions. NBER 11696, 2005.

BALANCE OF PAYMENTS AND INTERNATIONAL INVESTMENT POSITION MANUAL. International Monetary Fund, 2013.

Disponível em http://www.imf.org/external/pubs/ft/bop/2007/pdf/bpm6.pdf

BEKAERT, G.; Ehrmann, M.; Fratzscher, M.; Mehl, A. Global Crises and Equity Market Contagion. NBER Working Paper 17121, 2011.

BIS. "Capital flows and emerging market economies". Committee on the Global Financial System. CGFS Papers No 33, 2009.

BRONER, F.A.; Rigobon, R. Why are capital flows so much more volatile in emerging than in developing countries? 2004. Available at SSRN: http://ssrn.com/abstract $=884381$

BROTO, C.; Díaz-Cassou, J.; Erce, A. Measuring and explaining the volatility of capital flows to emerging countries. Journal of Banking \& Finance 35:1941-1953, 2011.

CALVO, G. "Capital flows and capital-markets crisis: the simple economics of sudden stops". Journal of Applied Economics, p. 35-54, Nov. 1998.

CALVO, G., Leiderman, L., Reinhart, C. "Capital Inflows and Real Exchange Rate Appreciation in Latin America: The Role of External Factors”. IMF Staff Papers 40 (1), 1993.

CALVO, G., Leiderman, L., Reinhart, C. "Inflows of Capital to Developing Countries in the 1990s". Journal of Economic Perspectives, American Economic Association, 10(2), pp: 123-139, 1996. 
CALVO, G.A., Izquierdo A., Mejia, L. On the Empirics of Sudden Stops: the Relevance of Balance-Sheet Effects, NBER 10520, 2004.

CALVO, G.A., Izquierdo A., Mejia, L. Systemic Sudden Stops: The Relevance of Balance-Sheet Effects and Financial Integration. NBER 14026, 2008.

CHICAGO BOARD OPTIONS EXCHANGE (2009). The CBOE VOLATILITY INDEX® - VIX $®$

COWAN, K.; De Gregorio, J.; Micco, A.; Neilson, C. "Financial diversification, sudden stops and sudden starts". In: Current Account and External Financing, edited by Kevin Cowan, Sebastián Edwards, and Rodrigo O. Valdés, Santiago, Chile. (C) 2008. Central Bank of Chile.

EDWARDS, S. Financial openness, sudden stops and current account reversals. Washington: NBER, 2004. (Working Paper, n. 10.277).

FAUCETTE, J.; Rothenberg, A; Warnock, F. Outflows-Induced Sudden Stops, The Journal of Policy Reform, 2005.Vol. 8, No. 2, 119-129.

FERNANDEZ-ARIAS, E. The new wave of private capital inflows: Push or pull? Journal of Development Economics 48, pp: 389-418, 1996.

FISHER, R. A. "On the interpretation of $\chi^{2}$ from contingency tables, and the calculation of P". Journal of the Royal Statistical Society 85 (1): 87-94, 1922.

FORBES K.; Warnock F. “Capital Flow Waves: surges, stops, flight and retrenchment”. NBER 17351, 2011.

FRATZSCHER, M. Capital Flows, Push versus Pull Factors and the Global Financial Crisis. Journal of International Economics 88. pp: 341-356, 2012.

GLOBAL FINANCIAL STABILITY REPORT. FINANCIAL MARKET TURBULENCE. CAUSES, Consequences, and Policies. "The Quality of Domestic Financial Markets and Capital Inflows" October 2007. International Monetary Fund.

GUIDOTTI, P. E.; Sturzenegger, F.; Villar, A. On the consequences of sudden stops. Economia, Spring, 2004.

INSTITUTIONAL OF INTERNATIONAL FINANCE. Capital Flows to Emerging Markets. May 2014.

LEVINE, R. Finance and Growth: Theory and Evidence. Handbook of Economic Growth, in: Philippe Aghion \& Steven Durlauf (ed.), Handbook of Economic Growth, edition 1, volume 1, chapter 12, pp. 865-934, 2005.

MORGAN, J.P. Emerging Markets Bond Index Plus (EMBI+) Rules and Methodology. J.P. Morgan Securities Inc. EmergingMarkets Research, 2004a.

MORGAN, J.P. EMBI Global and EMBI Global Diversified. Rules and Methodology. J.P. Morgan Securities Inc. EmergingMarkets Research, 2004b.

NEUMANN, R., Penl, R., Tanku, A. Volatility of capital flows and financial liberalization: do specific flows respond differently? International Review of Economics \& Finance 18(3). pp: 488-501, 2009.

ROCHA, K.; Moreira, A. A Volatilidade dos Fluxos de Capital para Economias Emergentes: O Papel da Qualidade Institucional do Governo e do Sistema Financeiro Doméstico. Texto para Discussão IPEA 1893, 2013.

SULA, O. Surges and Sudden Stops of Capital Flows to Emerging Markets. Open Economies Review, volume 21 (4), pp. 589-605, 2010.

WORLD ECONOMIC OUTLOOK. TOO SLOW FOR TOO LONG. "Understanding the Slowdown in Capital Flows to Emerging Markets". April 2016. International Monetary Fund. 


\section{Apêndice A}

As Tabelas Al e A2 apresentam os resultados e significância dos coeficientes estimados de determinantes de parada súbita, relaxando a amostra ao incluir período desde 1990 e total de 27 países emergentes. Os resultados permanecem, indicando robustez do modelo. O aumento da aversão ao risco global, das taxas de juros americanas (redução da liquidez) aumenta a probabilidade de ocorrência de parada súbita em todos os casos. Ainda, o aumento da capitalização bursátil e do crédito privado para fluxo total e IED diminuem a probabilidade de ocorrência de parada súbita.

Tabela Al - Determinantes de Parada Súbita - Amostra Pós 1990

\begin{tabular}{|c|c|c|c|c|c|c|c|c|c|}
\hline \multirow[t]{2}{*}{ Período Pós 1990} & \multicolumn{3}{|c|}{ Fluxo Líquido } & \multicolumn{3}{|c|}{ Carteira } & \multicolumn{3}{|c|}{ Investimento Externo Direto } \\
\hline & xtprobit & probit & xtlogit & xtprobit & probit & xtlogit & xtprobit & probit & xtlogit \\
\hline \multicolumn{10}{|l|}{ Variáveis Globais } \\
\hline VIX & $3.19^{\star *}$ & $2.81^{\star *}$ & $5.67^{\star \star}$ & $6.44^{\star \star}$ & $6.44^{\star *}$ & $11.08^{* *}$ & $4.25^{\star \star}$ & $4.24^{* *}$ & $7.23^{\star *}$ \\
\hline USTRY10 & 1.90 & 3.71 & -0.03 & $16.30^{* *}$ & $16.32^{* *}$ & $34.34^{\star *}$ & $15.83^{* *}$ & $15.77^{\star *}$ & $26.46^{\star *}$ \\
\hline \multicolumn{10}{|c|}{ Variáveis de Desenvolvimento do Sistema Financeiro Doméstico } \\
\hline Capitalização Bursátil & -0.26 & 0.12 & $-0.80^{\star *}$ & -0.25 & -0.16 & -0.30 & -0.23 & -0.22 & $-1.16^{\star \star}$ \\
\hline Crédito Privado & $-0.70^{\star *}$ & -0.35 & $-1.89^{\star \star}$ & $1.07^{\star *}$ & $0.56^{\star *}$ & $4.41^{\star *}$ & -0.11 & -0.12 & $-1.13^{* *}$ \\
\hline \multicolumn{10}{|l|}{ Variáveis Locais } \\
\hline Inflação & $0.05^{*}$ & 0.03 & $0.10^{\star *}$ & -0.04 & $-0.03^{*}$ & $-0.16^{* *}$ & -0.16 & $-0.15^{\star *}$ & -0.62 \\
\hline Juros Reais & 0.00 & 0.00 & 0.00 & -0.02 & $-0.02^{\star \star}$ & -0.04 & -0.07 & $-0.07^{\star \star}$ & 0.08 \\
\hline Abertura Comercial & $77.87^{\star \star}$ & $89.33^{* *}$ & 31.51 & -1.69 & -2.24 & 102.54 & $32.36^{\star \star}$ & $32.71^{* *}$ & -12.57 \\
\hline Crescimento PIB & $-4.04^{* *}$ & $-5.15^{\star \star}$ & $-6.20^{* *}$ & $2.61^{* *}$ & 2.29 & $4.92^{* *}$ & $-6.43^{* *}$ & $-6.41^{* *}$ & $-9.88^{* *}$ \\
\hline Reservas/Importação & 1.24 & 3.05 & 6.66 & $8.22^{*}$ & 6.69 & 7.57 & 3.08 & 2.74 & $31.68^{* *}$ \\
\hline
\end{tabular}

Fonte: Elaboração Própria. 
Tabela A2 - Determinantes de Parada Súbita - Amostra Total de 27 Emergentes

\begin{tabular}{|c|c|c|c|c|c|c|c|c|c|}
\hline \multirow[t]{2}{*}{ Emergentes Total } & \multicolumn{3}{|c|}{ Fluxo Líquido } & \multicolumn{3}{|c|}{ Carteira } & \multicolumn{3}{|c|}{ Investimento Externo Direto } \\
\hline & xtprobit & probit & xtlogit & xtprobit & probit & xtlogit & xtprobit & probit & xtlogit \\
\hline \multicolumn{10}{|l|}{ Variáveis Globais } \\
\hline $\mathrm{VIX}$ & $1.99^{\star *}$ & $1.77^{\star \star}$ & $3.47^{\star \star}$ & $5.23^{\star \star}$ & $5.09^{\star *}$ & $9.11^{* *}$ & $4.09^{\star \star}$ & $4.00^{* *}$ & $6.80^{* *}$ \\
\hline USTRY10 & -1.62 & 4.83 & -6.87 & $9.90^{* *}$ & 7.49 & $24.91^{* *}$ & $13.08^{* *}$ & $11.74^{* *}$ & $19.43^{* \star}$ \\
\hline \multicolumn{10}{|c|}{ Variáveis de Desenvolvimento do Sistema Financeiro Doméstico } \\
\hline Capitalização Bursátil & -0.24 & 0.19 & $-0.67^{\star *}$ & $-0.52^{\star *}$ & $-0.32^{*}$ & $-0.91^{* *}$ & $-0.33^{\star *}$ & $-0.21^{*}$ & $-1.27^{\star *}$ \\
\hline Crédito Privado & -0.24 & -0.12 & -0.59 & $1.27^{\star \star}$ & $0.57^{\star \star}$ & $3.37^{\star *}$ & 0.12 & 0.10 & -0.06 \\
\hline \multicolumn{10}{|l|}{ Variáveis Locais } \\
\hline Inflação & 0.01 & 0.00 & 0.02 & -0.05 & -0.02 & $-0.13^{\star *}$ & $-0.24^{*}$ & $-0.18^{* *}$ & -0.55 \\
\hline Juros Reais & 0.02 & 0.01 & 0.03 & -0.03 & $-0.03^{\star *}$ & -0.04 & -0.01 & -0.04 & 0.04 \\
\hline Abertura Comercial & $67.60^{* *}$ & $52.61^{* *}$ & $88.62^{* *}$ & 9.60 & 4.93 & $102.74^{\star}$ & 6.78 & 3.36 & -5.78 \\
\hline Crescimento PIB & $-3.70^{* *}$ & $-3.76^{\star \star}$ & $-5.97^{\star *}$ & 0.29 & -0.83 & 1.31 & $-4.47^{\star *}$ & $-3.94^{\star *}$ & $-7.63^{* *}$ \\
\hline Reservas/Importação & 3.32 & $11.60^{\star \star}$ & 4.20 & 6.18 & 4.09 & 11.01 & $9.20^{\star *}$ & 5.73 & $25.05^{\star *}$ \\
\hline
\end{tabular}

Fonte: Elaboração Própria. 